\title{
Concurrent Data Message Transmissions for Interference of Illegal Overhearing in Wireless Sensor Networks
}

\author{
Masahiro Okuri ${ }^{1, *}$, Hiroaki Higaki ${ }^{1,}{ }^{* *}$ \\ ${ }^{1}$ Department of Robotics and Mechatronics, Tokyo Denki University, Japan
}

\begin{abstract}
In wireless sensor networks, data messages containing sensor data achieved by a sensor module in a wireless sensor node is transmitted to a stationary wireless sink node along a wireless multihop transmission route in which wireless sensor nodes themselves forward the data messages. Each intermediate wireless sensor node broadcast data messages in its wireless transmission range to forward them to its nexthop intermediate wireless sensor node. Hence, eavesdropper wireless nodes within the wireless transmission range easily overhear the data messages. In order to interfere with the eavesdropper wireless nodes illegally overhearing the data messages in transmission, wireless sensor nodes whose wireless transmission ranges overlap and their next-hop intermediate wireless sensor nodes are out of the wireless transmission ranges each other forward data messages in transmission concurrently and cause collisions between these two data messages at any possible eavesdropper wireless nodes intentionally. To enhance regions where concurrently forwarded data messages intentionally collide to prevent their overhearing and to realize concurrent forwarding of data messages, this paper designes an algorithm for TDMA transmission slot assignments for more opportunities to interfere the eavesdropper wireless nodes.
\end{abstract}

\section{Introduction}

In wireless sensor networks, data messages containing sensor data achieved by sensor modules are transmitted from wireless sensor nodes to a stationary wireless sink node by wireless multihop transmissions. A data message forwarded by an intermediate wireless sensor node in a wireless multihop transmission route is broadcasted to all its neighbor wireless sensor nodes within its wireless signal transmission range. Hence, even though a neighbour wireless sensor node is not the next-hop neighbor wireless sensor node along the route, it can overhear the data message. Thus, an eavesdropper wireless node within the wireless signal transmission range can also overhear the data message. In order to solve this problem, an intentional collision between the data message and another one forwarded by another wireless sensor node whose wireless signal transmission range includes the eavesdropper wireless node prevents such illegal overhearing. This paper proposes a novel method to assign TDMA (Time Division Multiple Access) transmission slots to each wireless sensor node to interfere with overhearing of eavesdropper wireless nodes.

\section{Related Works}

In wireless multihop networks such as wireless ad-hoc networks and mesh networks, some methods for interfering with illegal overhearing of data messages by eavesdropper wireless nodes by transmitting noise

\footnotetext{
e-mail: okuri@higlab.net

e-mail: hig@higlab.net
}

messages have been proposed. In [2], for transmissions of data messages from a wireless node $N^{s}$ to $N^{d}$, Ns transmits the data messages to $N^{d}$ by using a directional antenna and $N^{d}$ transmits concurrently noise messages to all its neighbor wireless nodes by using an omni antenna. Since these messages collides at wireless nodes in the common region of wireless transmission ranges of the data messages and the noise ones, no wireless nodes receive pure data messages. One of the allied wireless nodes receiving the superimposed messages forwards them to $N^{d}$. Then, $N^{d}$ can remove the noise messages from it and get the data message since it knows the noise messages which $N^{d}$ itself transmitted. In order to apply this method in wireless sensor networks where all the wireless sensor nodes are required to forward data messages, all the wireless sensor nodes should possess both directional and omni antennas and enough processing power for signal processing to remove the noise messages from the superimposed messages. In [7], a method for transmitting noise messages from some 1-hop and 2-hop neighbour wireless nodes of an intermediate wireless node $N_{i}$ in a wireless multihop transmission route concurrently with the data messages from $N_{i}$ to its next-hop wireless node $N_{i+1}$ have been proposed. The concurrent transmissions cause intentional collisions at neighbor wireless nodes including eavesdropper ones. By selecting neighbor wireless nodes transmitting noise messages carefully, the data messages are surely received by $N_{i+1}$ without collisions. In this method, neighbor wireless nodes are required to transmit noise messages which has no contribution to transmit data messages and limited battery 
power in wireless sensor nodes is consumed.

For performance improvement in wireless sensor networks, some method introducing TDMA slot assignment have been proposed [1,3,5,6,8,9]. In [5], collision-free data message transmissions are realized by assigning different TDMA transmission stols to wireless sensor nodes whose wireless trasmission ranges contain one of their next-hop wireless sensor nodes. Here, the number of required TDMA transmission slots are reduced for improvement of data message transmission throughput.

\section{Proposal}

In [7], a method for interfering with the illegal overhearing of data messages by eavesdropper wireless nodes by intentional collisions between data messages and noise messages in wireless ad-hoc networks has been proposed. In order to make difficult for an eavesdropper wireless node to overhear a data message transmitted a wireless multihop transmission route and forwarded from an intermediate wireless node $N_{i}$ to its next-hop one $N_{i+1}$, wireless sensor nodes which are 1-hop neighbor ones of $N i$ and are not 1-hop neighbor ones of $N_{i+1}$ and which are common 2-hop neighbor ones of $N_{i}$ and $N_{i+1}$ transmit noise messages concurrently with the data message transmission to collide with the data message not at $N_{i+1}$ but at possible eavesdropper wireless nodes. However, transmissions of noise messages require additional transmission power consumption and might cause unnecessary collisions and contentions. Hence, this paper proposes a novel method for preventing illegal overhearing by intentional collisions in wireless sensor networks. Here, a cooperative approach by wireless sensor nodes with help of appropriate assignments of TDMA transmission slots to each wireless sensor node satisfies both of prevention of illegal overhearing by eavesdropper wireless nodes and avoidance of requirement for additional communication overheard. Under an assumption of a routing protocol with flooding of route request control messages issued by a stationary wireless sink node, assignments of TDMA transmission slots of data messages to wireless sensor nodes realize efficient intentional collisions to bother eavesdropper wireless nodes.

In order to formalize effect of concurrent transmissions of a data message and a noise message between a pair of neighbor wireless sensor nodes, the following relation between them is defined:

\section{[Assistable Wireless Sensor Nodes]}

Suppose $N_{i}$ and $N_{i+1}$ be an intermediate wireless sensor node and its next-hop one to transmit data messages to a stationary wireless sink node. A wireless sensor node $N$ is an assistable one for $N_{i}$ represented by $N \Rightarrow N_{i}$ if wireless transmission ranges of $N$ and $N_{i}$ overlap and $N_{i+1}$ is out of the wireless transmission range of $N$ as shown in Figure 1.

Satisfying $N \Rightarrow N_{i}$, if $N$ transmits a noise message to its neighbor wireless sensor node except for $N_{i}$ concurrently with $N_{i}$, it makes impossible for eavesdropper wireless nodes in a common region of the wireless transmission

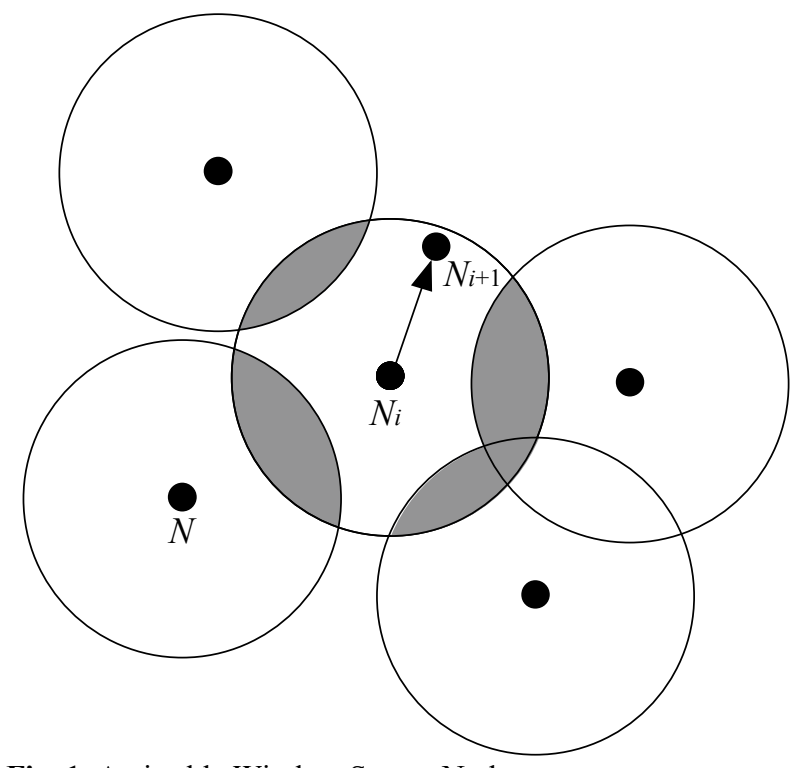

Fig. 1. Assistable Wireless Sensor Nodes.

ranges of $N$ and $N_{i}$ due to an intentional collision between the noise and the data messages concurrently transmitted by $N$ and $N_{i}$. Here, $N_{i+1}$ receives the data message from $N_{i}$ without a collision at $N_{i+1}$ with the noise message from $N$ since $N_{i+1}$ is out of the wireless transmission range of $N$.

Effect on avoidance of illegal overhearing of a data message by possible eavesdropper wireless nodes by the intentional collisions caused by concurrently transmitted a noise message can be evaluated by area of the common region of the wireless transmission ranges of $N$ and $N_{i}$. Hence, the following straight contribution for overhearing interference is introduced:

[Straight Contribution for Overhearing Interference] Straight contribution for overhearing interference for a wireless intermediate wireless sensor node $N_{i}$ by a wireless sensor node $N$ represented by $S C\left(N \Rightarrow N_{i}\right)$ is the ratio of area of the common region of the wireless transmission ranges of $N$ and $N_{i}$ to area of the wireless transmission range of $N_{i} . S C\left(N \Rightarrow N_{i}\right)=0$ if $N \nRightarrow N_{i}$ and $S C\left(N \Rightarrow N_{i}\right)>0$ otherwise. $\square$

In [7], for a data message transmission from $N_{i}$ to $N_{i+1}$, all 1-hop and part of 2-hop neighbor wireless sensor nodes $N$ satisfying $N \Rightarrow N_{i}$ transmits noise messages concurrently with the data message to interfere with illegal overhearing of the data message by possible eavesdropper wireless nodes in the common regions of wireless transmission ranges of $N_{i}$ and $N$ by intentional collisions. Here, the noise messages are transmitted only for causing the intentional collisions and the battery power consumption for the noise message transmissions does not contribute to other data message transmissions. In order for more efficient battery power consumption in wireless sensor nodes without any continuous power supply, $N$ transmits not a noise message but a data message to its next-hop intermediate wireless sensor node concurrently with $N_{i}$. Concurrent transmissions of the data messages by $N$ and $N_{i}$ contribute for both their transmission themselves and intentional collisions between them for avoidance of overhearing. In order to achieve this improvement, not only the data message from $N_{i}$ but also that from $N$ is also transmitted without 
collisions between them at the next-hop wireless sensor node of $N$. These requirements are formalized as the following mutual assistable wireless sensor nodes.

\section{[Mutual Assistable Wireless Sensor Nodes]}

If wireless sensor nodes $N_{i}$ and $N_{j}^{\prime}$ are mutually assistable wireless sensor nodes, i.e., both $N_{j}^{\prime} \Rightarrow N_{i}$ and $N_{i} \Rightarrow N_{j}^{\prime}$ are satisfied, $N_{i}$ and $N_{j}^{\prime}$ are mutual assistable wireless sensor nodes, which is represented by $N_{j}^{\prime} \leftrightarrow N_{i}$. $\square$

As shown in Figure 2, intermediate wireless sensor nodes $N_{i}$ and $N_{j}^{\prime}$ forward data messages to their next-hop wireless sensor nodes $N_{i+1}$ and $N^{\prime}{ }_{j+1}$, respectively, to transmit them to a stationary wireless sink node along wireless multihop transmission routes. Since wireless transmission ranges of $N_{i}$ and $N_{j}^{\prime}$ overlap, concurrent transmissions of data messages by $N_{i}$ and $N^{\prime}{ }_{j}$ cause intentional collisions at possible eavesdropper wireless nodes within the common region of the wireless transmission ranges which interfere their illegal overhearing of data messages. In addition, neither $N_{i+1}$ nor $N^{\prime}{ }_{j+1}$ are included in the wireless transmission ranges of $N_{j}^{\prime}$ or $N_{i}$, respectively. Hence, the data messages can be concurrently forwarded without mutual collisions at $N_{i+1}$ and $N_{j+1}^{\prime}$. As for straight contribution for overhearing interference, there is the following property:

[Property]

$\operatorname{SC}\left(N_{j}^{\prime} \Rightarrow N_{i}\right)=\operatorname{SC}\left(N_{i} \Rightarrow N_{j}^{\prime}\right)$ where $N_{j}^{\prime} \leftrightarrow N_{i}$.

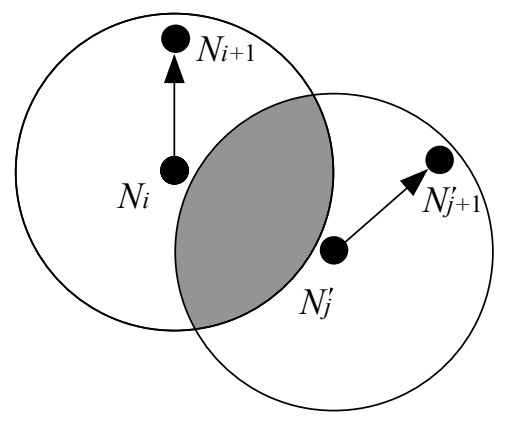

Fig. 2. Mutually Assistable Wireless Sensor Nodes.

In order for interfering with illegal overhearing by eavesdropper wireless nodes within the common region of wireless transmission ranges of intermediate wireless sensor nodes $N_{i}$ and $N_{j}{ }_{j}$ due to intentional collisions between data messages forwarded from $N_{i}$ and $N_{j}^{\prime}$ to $N_{i+1}$ and $N^{\prime}{ }_{j+1}$, respectively, the following two conditions are required to be satisfied: (1) Both $N_{i}$ and $N_{j}{ }_{j}$ have data messages to be forwarded to their next-hop intermediate wireless sensor nodes $N_{i+1}$ and $N^{\prime}{ }_{j+1}$, respectively. (2) Both $N_{i}$ and $N_{j}^{\prime}$ forward data messages concurrently, i.e., synchronous transmissions of data messages by $N_{i}$ and $N_{j}^{\prime}$ are realized.

In wireless sensor networks, each wireless sensor node $N_{i}$ forwards data messages initiated by itself, i.e., carrying sensor data achieved by its own sensor module, and ones initiated by other wireless sensor nodes and forwarded from its previous-hop wireless sensor nodes, i.e., carrying sensor data achieved by sensor modules in the other wireless sensor nodes. Some sensor modules achieve sensor data repeatedly with certain intervals and the others achieve sensor data according to occurrence of certain specific events. Hence, for avoidance of losses of data messages in transmission, each wireless sensor node possesses its buffer to store the data messages temporarily. Especially wireless sensor nodes near a stationary wireless sink node almost always hold some data messages in their buffer due to concentration of data messages in transmission. Hence, some method for avoidance of buffer overflow in intermediate wireless nodes in wireless ad-hoc networks have been discussed and proposed [4]. Therefore, there are many opportunities for both $N_{i}$ and $N_{j}^{\prime}$ satisfying $N_{i} \leftrightarrow N_{j}^{\prime}$ to have data messages in their buffer simultaneously.

In [7], synchronization between a data message transmission by an intermediate wireless node $N_{i}$ and noise message transmissions by its 1-hop and 2-hop neighbor nodes $N_{j}$ satisfying $N_{j} \Rightarrow N_{i}$ is realized by extended RTS/CTS control. In usual wireless multihop networks such as wireless ad-hoc networks, it is assumed that transmission requests of data messages are initiated not so frequently and wireless multihop transmission routes are detected in an on-demand manner for resilience to mobility of wireless nodes, lack of battery power and failure in wireless nodes. However, in wireless sensor networks, transmissions of data messages are continuously requested and it is required to avoid additional battery consumption for control message transmissions due to limited battery capacity in wireless sensor nodes. Hence, various methods to determine transmission schedule in each wireless sensor node in advance have been discussed and proposed [1, 3, 5, 6, 8, 9]. In this paper, each wireless sensor node possesses its closely synchronized local clocks and transmission slots are assigned to each wireless sensor node for realizing concurrent transmissions of data messages by mutually assistable wireless sensor nodes to interfere illegal overhearing by intentional collisions between these data messages.

In order to make more difficult for eavesdropper wireless nodes to overhear data messages forwarded from an intermediate wireless sensor node $N_{i}$ to its next-hop one $N_{i+1}$, it is required for more mutually assistable wireless sensor nodes $N_{j}^{\prime}$ of $N_{i}$ where $N_{i} \leftrightarrow N_{j}^{\prime}$ is satisfied to forward data messages concurrently, i.e., the same TDMA transmission slot is assigned to $N_{j}^{\prime}$ as $N_{i}$. Here, the data messages forwarded by multiple mutually assistable wireless sensor nodes of $N_{i}$ are also required to be forwarded to their next-hop wireless sensor nodes of $N_{j}{ }_{j}$ without collisions as shown in Figure 3. That is, in order to assign the same TDMA transmission slot to wireless sensor nodes $N_{i}, N^{\prime}{ }_{j}$ and $N^{\prime \prime}{ }_{k}$ where $N_{i} \leftrightarrow N^{\prime}$ and $N_{i} \leftrightarrow$ $N^{\prime \prime}{ }_{k}$ are satisfied for more secure data message forwarding from $N_{i}$ to $N_{i+1}$, data messages forwarded by $N_{j}{ }_{j}$ and $N^{\prime}{ }_{k}$ are required not to collide at neither $N^{\prime}{ }_{j+1}$ nor $N^{\prime \prime}{ }_{k+1}$, i.e., both $N^{\prime}{ }_{j+1}$ and $N^{\prime \prime}{ }_{k+1}$ are required to be out of the wireless transmission ranges of $N^{\prime \prime}{ }_{k}$ and $N_{j}{ }_{j}$, respectively.

This paper proposes a slot assignment method where a wireless sensor node to which a TDMA transmission slot has not yet assigned and to be a mutually assistable wireless sensor node with a 1-hop or 2-hop neighbor wireless sensor node to which a TDMA transmission slot has already been assigned is selected one by one and is assigned the same TDMA transmission slot to cause the 


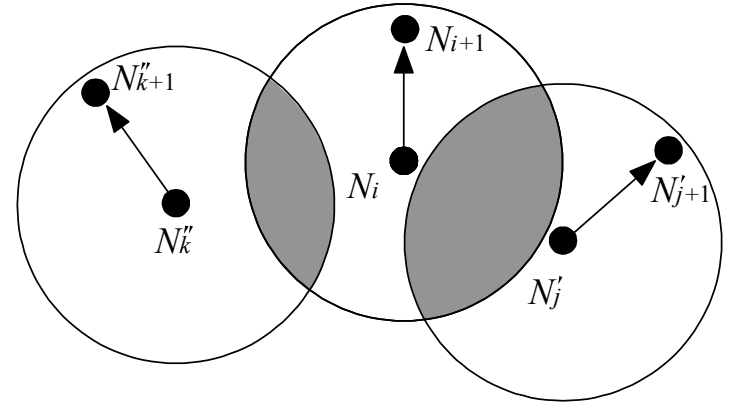

Fig. 3. Interference of Illegal Overhearing by Concurrent Data Message Forwarding by Multiple Mutually Assistable Wireless Sensor Nodes.

intentional collisions between concurrently forwarded data messages. In Figure 4, the same TDMA transmission slot has already been assigned to wireless sensor nodes $N_{l}$, $N_{2}, N_{3}$ and $N_{4}$ and one of the wireless sensor nodes which are mutually assistable wireless sensor nodes of one of these nodes is selected and is assigned the same slot.

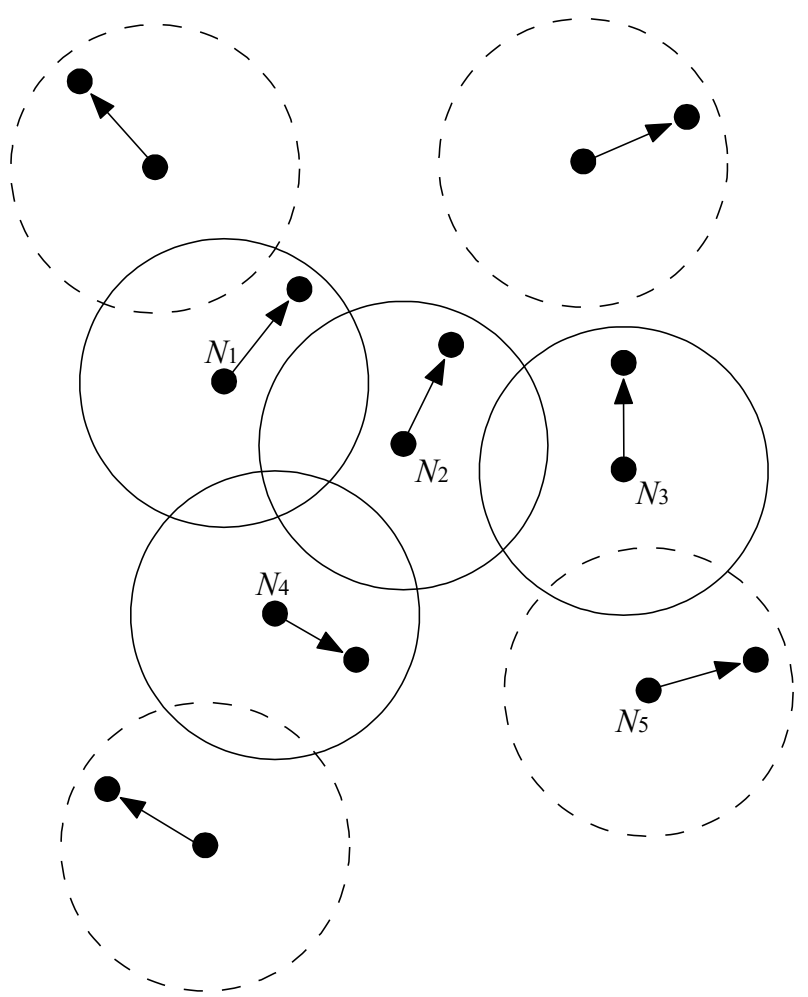

Fig. 4. Selection of Wireless Sensor Nodes for Assignment of Same Transmission Slot.

For example, a wireless sensor node $N_{5}$ is a mutually assistable one of $N_{3}$ and its next-hop wireless sensor node is out of wireless transmission ranges of $N_{l}, N_{2}, N_{3}$ and $N_{4}$. Hence, even if these 5 wireless sensor nodes forward data messages concurrently, i.e., in the same TDMA transmission slot, no collisions occur at their any next-hop wireless sensor nodes. Here, the area of overlap regions of their wireless transmission ranges of the wireless sensor nodes with the same TDMA transmission slot is increased by this additional slot assignment, which makes difficult for eavesdropper wireless nodes to overhear the forwarded data messages due to additional intentional collisions between the forwarded data messages.

For discussion about this incremental, i.e., one by one slot assignment method, a set of transitive mutually assistable wireless sensor nodes is defined:

\section{[Set of Transitive Mutually Assistable Wireless Sensor} Nodes]

Wireless sensor nodes $N$ and $N$ ' ' are transitive mutually assistable wireless sensor nodes if both $N \leftrightarrow N$ and $N^{\prime} \leftrightarrow \leftrightarrow$ $N^{\prime \prime}$ are satisfied, which is represented by $N \Leftrightarrow N$ '. In addition, $\operatorname{RMNS}(N)$ is a set of wireless sensor nodes which are transitive mutually assistable wireless sensor nodes of $N$, i.e., $\operatorname{RMNS}(N):=\left\{N^{\prime} \mid N \Leftrightarrow N^{\prime}\right\}$. $\square$

[Property]

$N \in \operatorname{RMNS}(N)$ is satisfied. $\square$

In our proposed method, one of the wireless sensor nodes which are mutually assistable wireless sensor nodes of one of the sensor nodes of a set $N S^{1}$ of ones to which the same TDMA transmission slot is assigned and whose next-hop wireless sensor nodes are not included in the wireless transmission ranges of the wireless sensor nodes in NS is selected and assigned the same TDMA transmission slot. Let $N^{\prime}$ be this selected wireless sensor node and $N \Leftrightarrow N$ ' is satisfied for $\forall N \in N S$.

An algorithm for assignment of TDMA transmission slots based on this method is designed. Here, this algorithm is greedy one since one of the multiple candidate wireless sensor nodes satisfying the conditions for assigning the same TDMA transmission slot is selected with the maximum straight contribution for overhearing interference. Hence, the selection is not always the best since the selection is not based on the additional contribution for overhearing interference. Even if there are no candidate mutually assistable wireless sensor nodes for assignment of the same TDMA transmission slot, the same slot can be assigned to wireless sensor nodes whose wireless transmission ranges do not overlap with those of the wireless sensor nodes to which the same TDMA transmission slot has already been assigned. Another TDMA transmission slot begins to be assigned only when it becomes impossible for any wireless sensor node to which no TDMA transmission slots have been assigned to be assigned the same TDMA transmission slot, i.e., any additional assignment of the same TDMA transmission slot causes collisions between data messages at one of the next-hop intermediate wireless sensor nodes.

\section{[Slot Assignment Algorithm]}

1. If TDMA transmission slots are assigned to all the wireless sensor nodes, the slot assignment algorithm terminates. Otherwise, one of the wireless sensor nodes $N$ to which no TDMA transmission slots have been assigned is randomly selected.

2. If there are TDMA transmission slots which have already been assigned to at least one of the wireless sensor nodes and have not yet assigned to any wireless sensor nodes satisfying $N^{\prime} \Rightarrow N$, one of such TDMA transmission slot is assigned to $N$.

3. For all the wireless sensor node $N^{\prime}$ which satisfies $N^{\prime}$ $\in \operatorname{RMNS}(N)$ and to which the same TDMA

\footnotetext{
${ }^{1}$ For $\forall N_{o} \in N S, N S \subset R M N S\left(N_{o}\right)$ is satisfied.
} 
transmission slot is assigned as $N$, the same TDMA transmission slot is assigned to a wireless sensor node $N^{\prime \prime}$ ' which satisfies $N^{\prime} \leftrightarrow N^{\prime \prime}$, and whose $\operatorname{SC}\left(N^{\prime}\right.$, $\left.\Leftrightarrow N^{\prime \prime}\right)$ is the maximum as $N$. If there are such wireless sensor node $N^{\prime \prime}$, skip to 3. Otherwise, skip to $1 . \square$

\section{Concluding Remarks}

This paper proposes a novel method to make difficult for eavesdropper wireless nodes to overhear data messages illegally in wireless sensor networks. For interfering the overhearing, 1-hop or 2-hop neighbor wireless sensor nodes concurrently transmits data messages which cause intentionally collisions at their common 1-hop neighbor wireless sensor nodes including eavesdropper wireless nodes and excluding their next-hop wireless sensor nodes. This concurrent transmissions of data messages for the intentional collisions are realized by assigning the same TDMA transmission slot. This paper proposes an algorithm realizing the slot assignments which enhance the area of common regions between wireless transmission ranges of wireless sensor nodes assigned the same TDMA transmission slot. As a result, the opportunities causing intentional collisions of data messages are increased and wireless sensor networks becomes more resilient to eavesdroppers. In future work, the authors evaluate our proposed slot assignment algorithm by simulation experiments to make clear that the algorithm provides more intentional collisions with a few additional numbers of slots in comparison with some conventional slot assignment algorithms.

\section{References}

1. Bo, Y., Jianzhong, L. and Yingshu, L., "Distributed Data Aggregation Scheduling in Wireless Sensor Networks," Proceedings of the 28th IEEE INFOCOM, pp. 2159-2161 (2009).

2. He, X. and Yener, A., "Two-Hop Secure Communication Using an Untrusted Relay: A Case for Cooperative Jamming," Proceedings of the IEEE Global Telecommunications Conference (2008).

3. Liqi, S. and Abraham, F., "TDMA Scheduling with Optimized Energy Efficiency and Minimum Delay in Clustered Wireless Sensor Networks," IEEE Transaction on Mobile Computing, vol. 9, no. 7, pp. 927-939 (2009).

4. Maurya, S., Gupta, V. and Jain, V.K., "LBRR: Load Balanced Ring Routing Protocol for Heterogeneous Sensor Networks with Sink Mobility," Proceedings of the Wireless Communications and Networking Conference (2017).

5. Ozlem, I., Amitabha, G., Bhaskar, K. and Krishnakant, C., "Fast Data Collection in Tree-Based Wireless Sensor Networks," IEEE Transactions on Mobile Computing, vol. 11, no. 1, pp. 86-99 (2012).

6. Rajendran, V., Obraczka, K. and Garcia-LunaAceves, J., "Energy-Efficient, Collision-Free Medium Access
Control for Wireless Sensor Networks," Journal of Wireless Networks, vol. 12, pp. 63-78 (2006).

7. Shimada, I. and Higaki, H., "Intentional Collisions for Secure Ad-Hoc Networks," Proceedings of the 13th International Conference on Mobile and Ubiquitous Systems, pp. 183-188 (2016).

8. Wen-Zan, S., Renjie, H., Behrooz, S. and Richard, L., "TreeMAC: Localized TDMA MAC Protocol for Real-time High-data-rate Sensor Network," Pervasive and Mobile Computing, vol. 5, pp. 750 765 (2009).

9. Yanwei,W., Xiang-Yang, L., YunHao, L. andWei, L., "Energy-Efficient Wake-Up Scheduling for Data Collection and Aggregation," IEEE Transaction on Parallel and Distributed System, vol. 21, no. 2, pp. 275-287 (2010). 